Article

\title{
Genome-Wide Identification, Characterization and Expression Analysis of Xyloglucan Endotransglucosylase/Hydrolase Genes Family in Barley (Hordeum vulgare)
}

\author{
Man-Man Fu ${ }^{1}$, Chen Liu $^{1}$ (D) and Feibo $\mathrm{Wu}^{1,2, *}$ \\ 1 Department of Agronomy, College of Agriculture and Biotechnology, Zijingang Campus, Zhejiang University, \\ Hangzhou 310058, China; sunshineabigail@163.com (M.-M.F.); liu-chen@zju.edu.cn (C.L.) \\ 2 Jiangsu Co-Innovation Center for Modern Production Technology of Grain Crops, Yangzhou University, \\ Yangzhou 225009, China \\ * Correspondence: wufeibo@zju.edu.cn; Tel./Fax: +86-571-8898-2827
}

Academic Editors: Quan Zou, Xiangxiang Zeng and Alfonso Rodríguez-Patón Received: 1 April 2019; Accepted: 17 May 2019; Published: 20 May 2019

\begin{abstract}
Xyloglucan endotransglucosylase/hydrolases (XTHs) —a family of xyloglucan modifying enzymes-play an essential role in the construction and restructuring of xyloglucan cross-links. However, no comprehensive study has been performed on this gene family in barley. A total of $24 \mathrm{HvXTH}$ genes (named HvXTH1-24) and an EG16 member were identified using the recently completed genomic database of barley (Hordeum vulgare). Phylogenetic analysis showed that 24 HvXTH genes could be classified into three phylogenetic groups: (I/II, III-A and III-B) and HvXTH15 was in the ancestral group. All HvXTH protein members-except HvXTH15-had a conserved $\mathrm{N}$-glycosylation site. The genomic location of $\mathrm{HvXTHs}$ on barley chromosomes showed that the 24 genes are unevenly distributed on the 7 chromosomes, with 10 of them specifically located on chromosome $7 \mathrm{H}$. A structure-based sequence alignment demonstrates that each XTH possesses a highly conserved domain (ExDxE) responsible for catalytic activity. Expression profiles based on the barley genome database showed that $\mathrm{HvXTH}$ family members display different expression patterns in different tissues and at different stages. This study is the first systematic genomic analysis of the barley $\mathrm{HvXTH}$ gene family. Our results provide valuable information that will help to elucidate the roles of $\mathrm{HvXTH}$ genes in the growth and development of barley.
\end{abstract}

Keywords: Xyloglucan endotransglucosylase/hydrolase (XTH); genome-wide; gene expression; barley

\section{Introduction}

Xyloglucan endotransglucosylase/hydrolases (XET/XEHs also named XTHs) are a family of xyloglucan modifying enzymes that have two different catalytic activities and can act either as endotransglucosylases (XET, EC 2.4.1.207) or as endohydrolases (XEH, EC 3.2.1.151). XTHs belong to glycoside hydrolase family 16 and play an important role in the construction and restructuring of xyloglucan cross-links [1]; their evolution has recently been studied. Faure et al. [2] found some enzymes, such as GH16 XETs and xyloglucanases, may have the ability to catalyze hetero-transglycosylation reactions in nasturtium (Tropaeolum majus) and the unique specificity of hetero-trans- $\beta$-glucanase (HTG) in horsetails evolved from XTH and is due to three key amino acid substitutions [3]. A previous analysis has indicated that XTHs are closely related to bacterial licheninases (EC 3.2.1.73), which specifically hydrolyze $\beta(1 \rightarrow 4)$ linkages [4]. A later study showed that a newly identified subfamily of GH16 endo- $\beta$-glucanases from plants are an evolutionary link between bacterial licheninases and XTH genes [5]. An enzymological characterization and X-ray crystallography of EG16 
from Vitis vinifera have also given a crystallographic insight into the evolutionary origins of XTHs [6]. Genomic and transcriptomic analysis revealed that EG16 represents an early adoption of the $\beta$-jellyroll scaffold from an early GH16 member, which then, diversified by loop deletion and the extension of $C$ terminus in XTHs [7].

The first systematic research of XTH gene family in A. thaliana showed that the XTH genes could be classified into three groups (I, II and III) [8,9]. A later study in rice (Oryza sativa) showed that there was no significant boundary between group I and II and the XTH genes in rice were classified into two major subgroups named I/II and III [10]. Based on the three-dimensional structures of Tm-NXG1 and Tm-NXG2 from nasturtium, XTHs in group III can be separated into two main clades (group III-A and group III-B) [11]. Furthermore, the study also gained a small outlier group (named ancestral group) close to the root of the tree. Detailed three-dimensional structures of Tm-NXG1 and Tm-NXG2 revealed the major structural features of the active site regulating relative rates of hydrolysis to transglycosylation in GH16 xyloglucan-active enzymes transformation, suggesting that XTH genes evolved to have a role in cell wall remodeling [11]. A study of XTHs in Fragaria vesca also suggested a similar classification of XTH genes and revealed differing expression levels of XTHs during different developmental stages of fruit ripening and in different tissues [12].

XTH genes play an active role in response to plant hormones. The expression of OsXTH8 was upregulated by gibberellic acid (GAs) and there was little response to other hormones [13]. Members in the XTH gene family respond differently to different plant hormones and no close correlation was found between the tissue-specific expression and the responses to plant hormones of XTH genes in A. thaliana [8]. For example, the XTH genes-AtXTH17, AtXTH18, AtXTH19 and AtXTH20-are phylogenetically closely related to each other and are preferentially expressed in roots but exhibit different expression profiles within roots and in response to hormonal signals [14]. Auxin and brassinosteroids (BR) can regulate a subset of XTHs during cell elongation [15]. AtXTH19 and AtXTH20 can be induced by ANAC071 (Arabidopsis NAC domain containing protein 71) in the distal part of an incised stem and were involved in cell proliferation in the tissue reunion process [16]. Expression of DkXTH6 was up-regulated during ethylene production, as well as by propylene and abscisic acid (ABA) treatments but suppressed by GA3 and a cold treatment, while DkXTH7 exhibited its highest transcript levels in GA3-treated fruit and cold-treated fruit [17].

With the development of Next Generation Sequencing (NGS), multigene families of XTHs have been reported both in monocotyledonous and dicotyledonous plants. There are 33 XTH family members in Arabidopsis thaliana [8], 29 in rice (Oryza sativa) [10], more than 57 in wheat (Triticum aestivum) [18], 35 in sorghum (Sorghum bicolor) [19], 56 in tobacco (Nicotiana tabacum) [20], 61 in soybean (Glycine max) [21], 21 in tomato (Solanum lycopersicum) [22,23], 41 in poplar (Populus spp.) [24]. Barley (Hordeum vulgare), one of the earliest domesticated species in the Near East, is the fourth most major cereal in the world [25]. Barley has been chosen for study, as it is an important crop species, as well as a model for genetic and physiological studies and breeding [26]. However, little is known about barley XTHs, except for the report that the expression of XET-related genes was related to elongation in leaves of barley [27] and can be heterologous expressed in Pichia pastoris [28]. Now the availability of barley genome and the comparative study of the XTH gene family across plant species provide an opportunity to explore the XTH diversity in barley [29,30]. In this study, we identified 24 candidate HvXTHs based on the bioinformatic analysis with the barley genome. Phylogenetic analysis indicated that the HvXTHs can be classified into three groups (I/II, III-A and III-B) and a small ancestral group. The structure and chromosomal location of $\mathrm{HvXTH}$ gene family members were also analyzed. Expression analysis showed that $\mathrm{HvXTHs}$ had different expression levels at different stages of development. HvXTH8 showed high expression in all tissues except in senescing leaves. The transcripts of HvXTH17 and HvXTH18 in different tissues were at the similar level. 


\section{Results and Discussion}

\subsection{Identification of $\mathrm{HvXTHs}$}

With the publication of barley genome, it is possible to study the XTH family in barley on a genome-wide level. EXT (GenBank: X91659.1), reported as a candidate HvXTH of barley, was filtered in this study because of a lack of a complete ORF [24]. A total of 24 candidate HvXTHs were identified in the barley genome using data from EnsemblPlants. The details of information relating to previously reported HvXTHs is presented in Table 1 and renamed 24 predicted HvXTHs based on chromosome position. Previous research showed that the number of XTHs in commelinid monocots was approximately 30 homologs per genome with more than 30 XTHs being found in maize, wheat and rice [7]; fewer XTH members were identified in barley. Moreover, an EG16 homolog (Gene ID: HORVU4Hr1G085940.1, Gene locus: chr4H: 633,032,657-633,033,820) was also identified [7], which appears to be the evolutionary link between bacterial licheninases and XTH gene products $[5,7]$.

Table 1. XTH genes identified in barley genome.

\begin{tabular}{ccccc}
\hline Gene Name & Previous Gene Name & \multicolumn{1}{c}{ Gene ID } & UniProtKB/TrEMBL & Chromosome \\
\hline HvXTH1 & HvPM5 [27] & HORVU1Hr1G087320.1 & - & chr 1H \\
HvXTH2 & & HORVU2Hr1G014530.3 & A0A287H550 & chr 2H \\
HvXTH3 & & HORVU2Hr1G045330.4 & M0W9B4 & chr 2H \\
HvXTH4 & & HORVU2Hr1G101160.2 & M0YQG6 & chr 2H \\
HvXTH5 & & HORVU3Hr1G016800.1 & F2D9R7 & chr 3H \\
HvXTH6 & & HORVU3Hr1G016820.3 & - & chr 3H \\
HvXTH7 & & HORVU3Hr1G016850.3 & A0A287K963 & chr 3H \\
HvXTH8 & & HORVU4Hr1G028720.1 & A0A287NP77 & chr 4H \\
HvXTH9 & & HORVU4Hr1G064220.1 & A0A287PD07 & chr 4H \\
HvXTH10 & XET7 [28] & HORVU4Hr1G090820.2 & F2DPR1 & chr 4H \\
HvXTH11 & & HORVU5Hr1G060340.3 & A0A287RFA0 & chr 5H \\
HvXTH12 & & HORVU6Hr1G067470.2 & A0A287U199 & chr 6H \\
HvXTH13 & & HORVU6Hr1G081590.1 & A0A287UX29 & chr 6H \\
HvXTH14 & HvPM2 [27] & HORVU6Hr1G093230.2 & F2D903 & chr 6H \\
HvXTH15 & & HORVU7Hr1G039600.2 & A0A287W560 & chr 7H \\
HvXTH16 & & HORVU7Hr1G086890.3 & M0XPV0 & chr 7H \\
HvXTH17 & & HORVU7Hr1G098260.1 & F2D1T0 & chr 7H \\
HvXTH18 & & HORVU7Hr1G098280.1 & A0A287XI65 & chr 7H \\
HvXTH19 & & HORVU7Hr1G098320.3 & A0A287XI88 & chr 7H \\
HvXTH20 & & HORVU7Hr1G098330.4 & - & chr 7H \\
HvXTH21 & HvXET6[31] & HORVU7Hr1G098370.1 & F2DM52 & chr 7H \\
HvXTH22 & & HORVU7Hr1G098390.1 & F2D337 & chr 7H \\
HvXTH23 & HvXEB [27] & HORVU7Hr1G098440.2 & F2DUU5 & chr 7H \\
HvXTH24 & & HORVU7Hr1G106530.2 & - & chr 7H \\
\hline
\end{tabular}

\subsection{Phylogenetic Analysis of HvXTHs}

A phylogenetic analysis was conducted with a total of 88 full-length protein sequences, using PhyML to study the evolutionary relationships of the XTH genes from barley $(H v X T H)$, rice $(O s X T H)$ and A. thaliana (AtXTH) (sequence information is provided in Supplementary File 1). The phylogenetic tree consists of three predominant branches with significant bootstrap support and used the structurally characterized bacterial lichenase (1GBG, EC 3.2.1.73) as an outgroup (Figure 1). HvXTH genes were classified into three groups: 18 genes in Group I/II, 2 in Group IIIA and 3 in Group IIIB. The ancestral group close to the root included AtXTH1, AtXTH2, AtXTH3, AtXTH11 and HvXTH15; this structure is similar to found in previous research [11]. It was reported that the merging of group I and II constructed the largest branch [10]. There was no significant difference between group I and group II which constituted the largest cluster and is referred to as group I/II (containing 22 AtXTHs, 18 OsXTHs and $18 \mathrm{HvXTHs);} \mathrm{this} \mathrm{result} \mathrm{is} \mathrm{also} \mathrm{consistent} \mathrm{with} \mathrm{a} \mathrm{previous} \mathrm{study} \mathrm{[10].} \mathrm{Three} \mathrm{HvXTH} \mathrm{genes} \mathrm{(HvXTH9,}$ 
HvXTH11 and HvXTH12) were classified into group III-B (including 5 AtXTHs and 7 OsXTHs) and HvXTH15 was in the ancestral group; thus, most genes may exhibit XET activity. Two members (HvXTH10 and HvXTH16) were in Group III-A and may have mixed glucosylase/hydrolases holding functions [11]. It seems that barley contains more XTHs in group I/II compared with rice. In dicotyledons, members of Group III have been shown to catalyze xyloglucan hydrolysis (xyloglucan endohydrolase activity) rather than xyloglucan transglucosylation (XET activity) [11,32,33]. A previous study of heterologous expression in the yeast Pichia pastoris [28] showed that HvXTH21 and HvXTH23 had detectable XET activity.

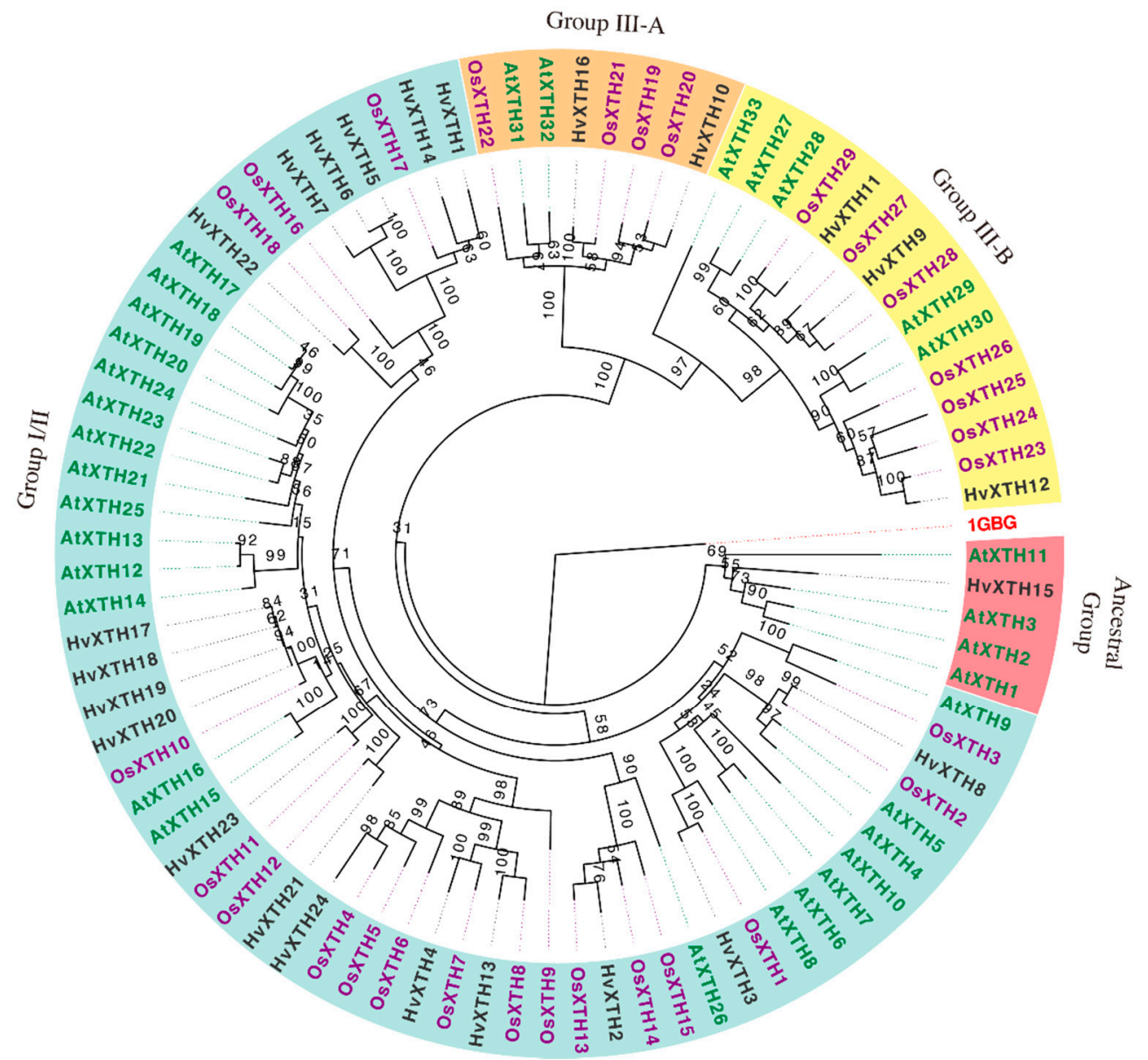

Figure 1. Phylogenetic tree of full-length HvXTH, OsXTH and AtXTH proteins. The tree was constructed using identified $24 \mathrm{HvXTHs}$ in barley, 33 AtXTHs from Arabidopsis and 29 OsXTHs from rice. The numbers at each fork of the tree indicate the number of times (expressed as percentage) that the group of genes was clustered together in 100 bootstrap replicates.

\subsection{Gene Structure Analysis and Chromosomal location}

To obtain further insight into the structural diversity of the XTH genes in barley, the structure of the $24 \mathrm{HvXTHs}$ (Figure 2) was identified using GSDS; coding and genomic sequences of HvXTHs are provided in Supplementary Files 2 and 3. The conserved motif sequence ExDxE was found in all HvXTH members (Figure 2). The number of exons varied from two to four which is similar to a previous study in rice [10]. The motif was always located on the second exon but also on the third exon of XTH (HvXTH3) in group I/II which is not consistent with study in $A$. thaliana [8,34]. SignalP was used to predict the presence of a signal peptide for entry into the secretory pathway for the 24 HvXTH proteins. The result showed that $22 \mathrm{HvXTH}$ proteins contained putative signal peptides which were all located in the first exon (Figure 2). 


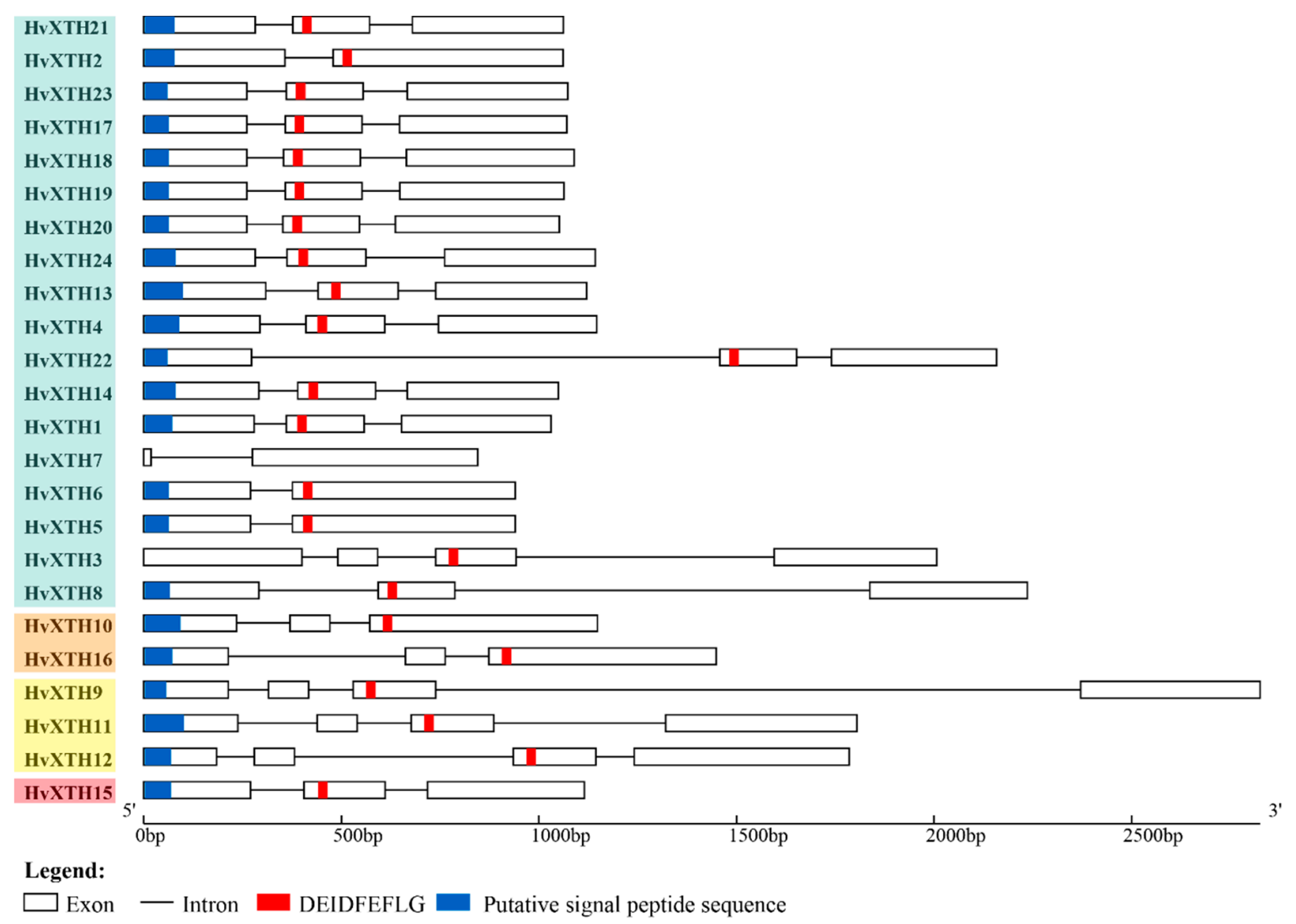

Figure 2. Gene structure and conserved protein domains of XTH genes in barley showing exons, introns, putative signal peptide sequences and motif sequence organization. Group I/II HvXTHs are in light blue, III/A in orange, III/B in yellow and the ancestral group in red. Sequence information is provided in Supplementary Files 3 and 4.

The chromosomal positions of $\mathrm{HvXTH}$ gene family members were located using information derived from the barley genome [29]. A total of $24 \mathrm{HvXTHs}$ were mapped to the genome (Figure 3). The HvXTH genes were unevenly distributed in the genome of the barley cultivar Morex. There was only one $\mathrm{HvXTH}$ gene located on chromosome $1 \mathrm{H}$ and chromosome $5 \mathrm{H}$. Three $\mathrm{HvXTHs}$ could be found on each of chromosomes $2 \mathrm{H}, 3 \mathrm{H}, 4 \mathrm{H}, 6 \mathrm{H}$. Chromosome $7 \mathrm{H}$ carried $10 \mathrm{HvXTHs}$ which was the maximum number. Five or fewer genes located within $100 \mathrm{~kb}$ were usually considered as tandem duplicates [6]. In this study, four gene pairs (HvXTH6/7, HvXTH17/18, HvXTH19/20, HvXTH21/22) were detected within $100 \mathrm{~kb}$ on chromosome $3 \mathrm{H}$ and chromosome $7 \mathrm{H}$, which may be the result of tandem duplication (Figure 3). The Smith-Waterman algorithm (http://www.ebi.ac.uk/Tools/psa/) suggested that the similarities of sequence between two pairs of genes (HvXTH17/18, HvXTH19/20) exceeded $90 \%$. Taken together, these two pairs are considered as tandem duplicates. Detailed information is shown in Supplementary File 4. According to the phylogenetic tree, HvXTH6 and HvXTH7 and HvXTH17/18/19/20 are closely related, which indicates that the gene duplications of these three pair of genes might be related to the evolution. 

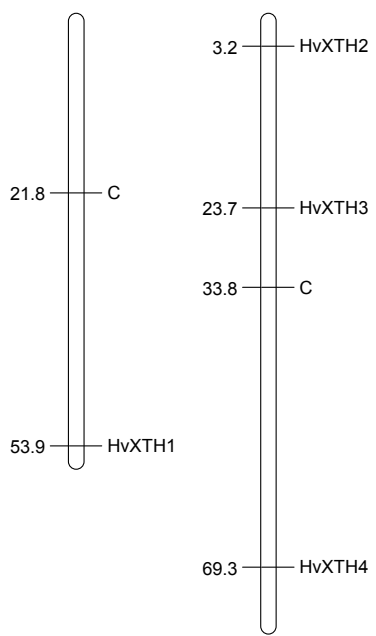
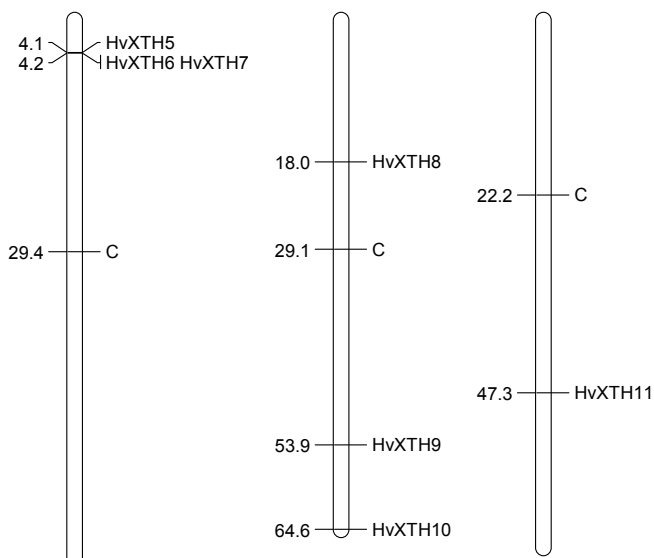


Figure 3. Distribution of $H v X T H$ genes in barley chromosomes. The location of $H v X T H$ gene family members in the genome barley cultivar 'Morex'. C indicates the centromere.

\subsection{Structure-Based Sequence Alignment}

The alignment of the $24 \mathrm{HvXTHs,} \mathrm{together} \mathrm{with} \mathrm{a} \mathrm{xyloglucan} \mathrm{endotransglycosylase-PttXET16-34}$ (PDB id: 1UN1) [35]—and a xyloglucan endohydrolase-TmNXG1 (PDB id: 2UWA) [11]—with known protein structures were used to predict the secondary structures of the HvXTH proteins of barley using ESPript (http://espript.ibcp.fr/ESPript/ESPript/). The HvXTHs in group I/II and group III had similar structures to PttXET16-34 and TmNXG1, respectively and the most conserved domains are shown in Figure 4. The entire alignment is provided in Supplementary Files 5 and 6 . All the members of the HvXTH protein possessed the XET/XEH C-terminal extension, while the endo-glucanases (EG16 members), which are the evolutionary link between bacterial lichenases and XTHs, normally lack this domain $[5,6]$.

The active site (ExDxE) containing the residues responsible for catalytic activity was highly conserved in all of the HvXTH family members. The first glutamate residue (E) acts as catalytic nucleophile which initiates the enzymatic reaction and the second one as a base to activate the substrate. All members of HvXTH family, except HvXTH15 (ancestral group), had a conserved $\mathrm{N}$-glycosylation site (marked with asterisks), which is proximal to the catalytic residues as reported in Fragaria vesca [12]. The $N$-glycosylation site domain $(\mathrm{NxT} / \mathrm{S} / \mathrm{Y})$ is thought to play a role in protein stability. Heterologous expression in Pichia pastoris produced some mannosylated protein glycoforms, which did not significantly affect enzymic activity [36].

Group IIIA had longer Loop 2 than the other groups and mainly showed xyloglucan hydrolase activity and previous structural and biochemical analysis of TmNXG indicated that the extension of loop 2 was a key factor in determining endo-xyloglucanase activity in XTH gene products [11]. There were no Ser-213 and Asn (Glu)-218 substitutions in group III-A; this result differs from the study in nasturtium, in which the substitutions were found in most sequences of group IIIA [11]. Members of group III-B (HvXTH9, HvXTH11 to HvXTH12) containing Ser (Ala)-213 substitutions and three residues are missing in loop3 which indicates they might have endotransglucosylase as their major enzyme activity [5-7]. The alignment of the EG16 protein encoded by HORVU4Hr1G085940.1 (Gene locus: chr4H: 633,032,657-633,033,820) with other representative EG16s from poplar, grape and selected monocot EG16s was also performed [5-7]. The analysis showed that EG16 and HvXTH share the same catalytic site. The full alignment is provided in Supplementary File 7. 

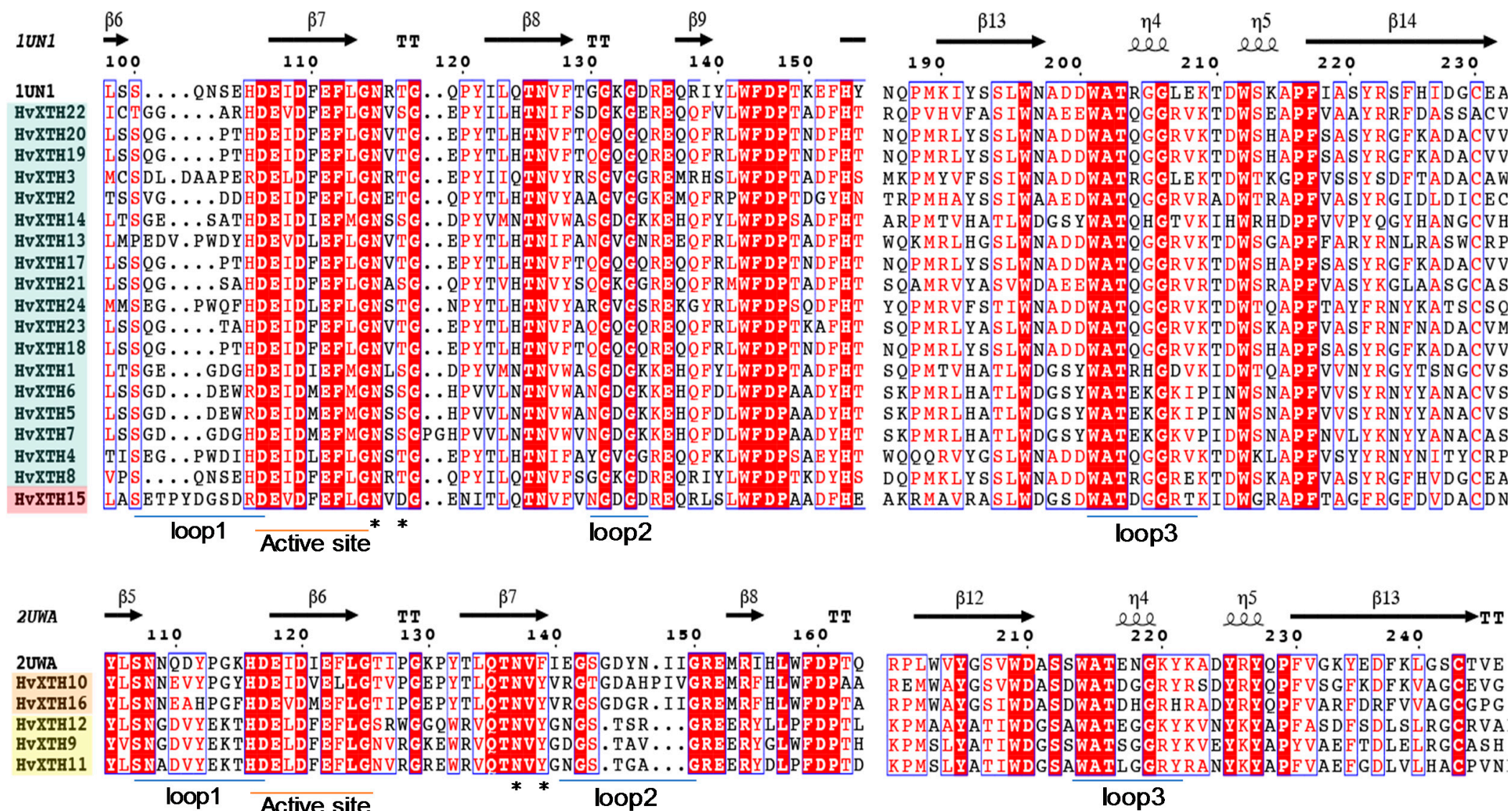

Figure 4. Structure-based sequence alignment of HvXTHs. Sequence were aligned using MEGA7 and secondary structures predicted using ESPript. HvXTHs in group I/II and group III had similar structures to PttXET16-34 (1UN1) and TmNXG1 (2UWA), respectively, whose structures have been experimentally determined. Group I/II HvXTHs are in light blue, group III/A in orange, group III/B in yellow, the ancestral group in red. Blue frames indicate conserved residues, white letters in red boxes indicate strict identity and red letters in white boxes indicate similarity. The secondary structures of $\beta$ sheets (arrows) and $\alpha$-helices (spiral) and loops 1,2 and 3 (lines) are indicated. $N$-glycosylation residues are indicated as asterisks. The entire alignment is presented in Supplementary Files 5 and 6. 


\subsection{Expression Patterns of HvXTHs at Different Developmental Stages and Tissues}

Transcript analysis showed that members of the HvXTH gene family had distinctly different expression patterns during different developmental stages as well as in different plant tissues (Figure 5; Supplementary File 8: Table S2). Some members of HvXTH (such as HvXTH8, HvXTH10, HvXTH14, HvXTH17, HvXTH18, HvXTH20 and HvXTH21) were expressed in almost all of the sixteen stages or tissue analyzed in the present study. Among them, HvXTH8 showed high expression in all tissues, especially in leaf tissue (ETI) and the third stem internode (NOD). HvXTH10 is mainly expressed in the rachis (RAC), lemma (LEM) and palea (PAL). The transcript level of HvXTH14 in caryopsis (CAR15) was higher than in other tissues. The expression pattern of HvXTH17 and HvXTH18 showed a high degree of similarity which indicates that they may play similar roles in the development of the plant. HvXTH20 showed a higher expression level in the lodicule (LOD), LEM and PAL compared with other tissues. HvXTH21 was highly expressed in NOD and LOD. While HvXTH2, HvXTH4, HvXTH9, HvXTH12, HvXTH15 and HvXTH23 showed lower expression in almost all developmental stages and tissues. Other members of the $\mathrm{HvXTH}$ family showed tissue-specific expression patterns. For example, the expression of $\mathrm{HvXTH} 24$ was only obviously detected in etiolated leaves (ETI) and roots (ROO2). HvXTH6 was only expressed in embryos (EMB), ROO, ROO2 and NOD. The transcript of $\mathrm{HvXTH7}$ was mainly expressed in roots (ROO and ROO2). HvXTH11 was almost only expressed in the developing inflorescence tissues, INF1 and INF2, which indicates that HvXTH11 might be involved in the inflorescence development. Both HvXTH19 and HvXTH22 showed a higher expression level in inflorescence tissues such as LOD, LEM and PAL.

Previous research has reported that only a few genes in the XTH family have been verified as encoding true XTH proteins [37,38]. Domain and structural analysis, together with the RNA-seq data, strongly indicated that these genes encode proteins. Previous research on AtXTH expression using GUS staining in Arabidopsis showed that AtXTHs are probably expressed in all developmental stages from seed germination through to flowering [39]. Consistent with these results, HvXTH family members play different roles in plant development. About half of the HvXTH family members showed higher expression in embryonic tissue from germinating grains (EMB), which indicates that HvXTHs might play an active role in seed germination and Aubert et al. showed that AtXTH31 is predicted to play a role in seed germination [40]. Almost all members of the HvXTH family are expressed in roots and leaves. Previous research has shown that AtXTH31 might be involved in root morphogenesis [39,40] and that AtXTH32 is primarily expressed in the shoot apex [39]. The location of LeEXT in epidermal cells in the elongating region suggests a role in elongation induced by auxin [41]. HvXTH might also play an essential role in this process.

Internode extension is related to plant height which is an essential element for crop breeding. Gene expression analysis showed that $\mathrm{HvXTH} 21$ has the highest expression in the third stem internode compared with other tissues as well as in comparison with all other members of the HvXTH family. These results indicate that members of $H v X T H$ family might affect the growth of barley through affecting stem extension. HvXTH20 shows the highest expression in LOD, LEM and PAL (which are significant components of inflorescence tissues) compared with other tissues. This indicates that higher expression of $\mathrm{HvXTH} 20$ may be beneficial for the development of inflorescence tissues (such as the extension of the rachis and lodicule) which might play an important role in seed formation. XTH activity in vegetative tissues was examined and showed that XTHs might be involved in stopping elongation, which indicates that the activity of XTHs is not always related to plant growth [42-44]. Previous study reported that the linkages between the G-layer and secondary cell walls are XET-dependent and have been implicated in transmitting tensile stresses [44,45].

Two grain developmental stages were used to analyze the expression patterns of the $24 \mathrm{HvXTH}$ family members. The result indicates that the expression of HvXTH8, HvXTH10, HvXTH14. HvXTH20 might play a role in the development of barley grain. The various expression patterns of $H v X T H$ family members in different developmental stages indicated that HvXTH genes play an active role in the development of barley [44]. 


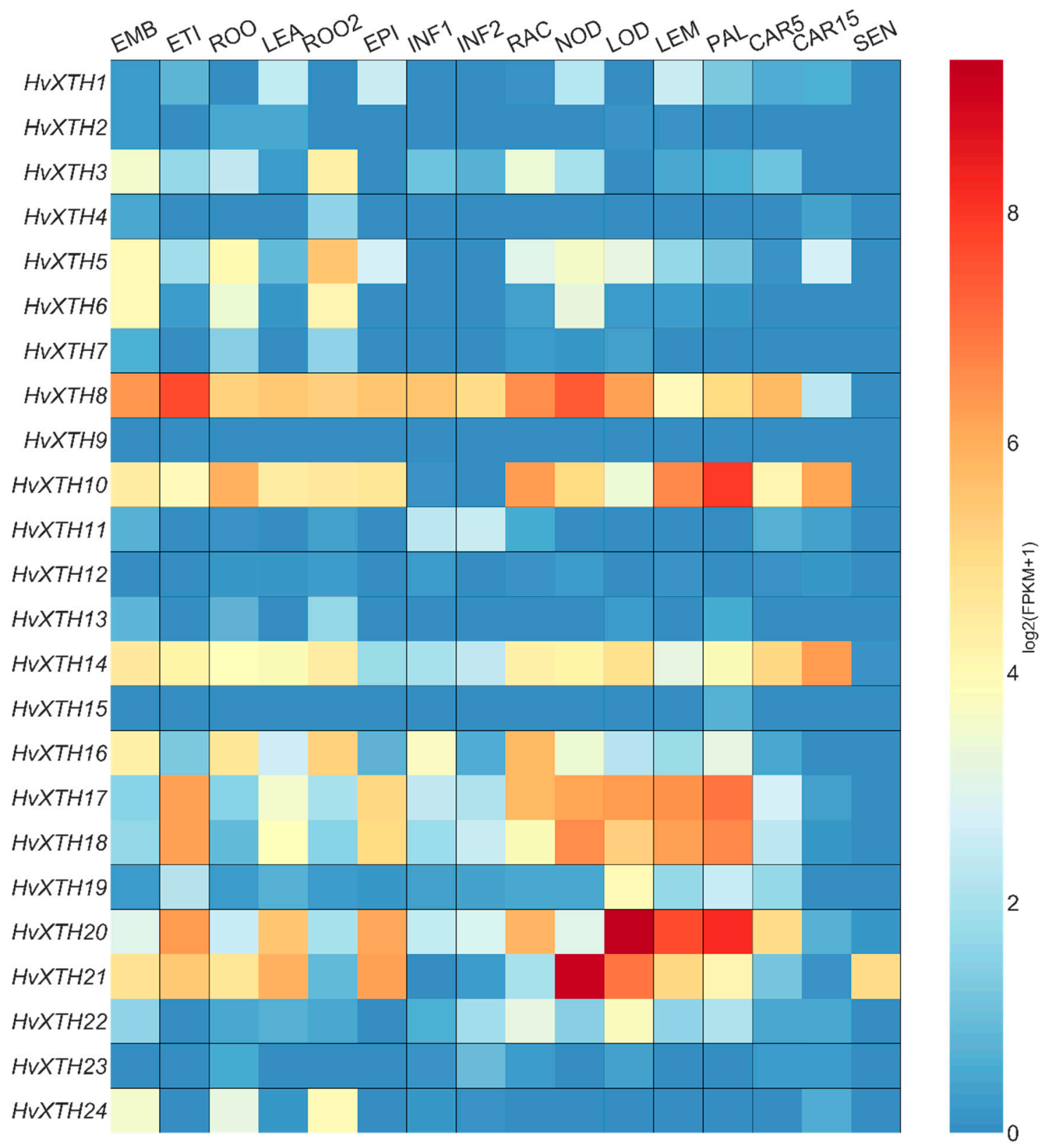

Figure 5. Heatmap of HvXTH gene expression in different tissues and stages of development. Data were obtained from a publicly available database, ENA (Accession number: PRJUEB3149, PRJEB14349). Rows represent $H v X T H$ members, while columns show different developmental stages and tissues. The expression level of $\mathrm{HvXTHs}\left(\log _{2}(\mathrm{FPKM}+1)\right)$ is shown by the intensity of color. $\mathrm{EMB}$, embryonic tissue; ETI, isolate etiolated leaf; ROO, root tissue (17 days and 28 days after planting); LEA, leaf tissue; EPI, epidermal strips; INF, whole developing inflorescence tissue (30 days and 50 days after planting); RAC, rachis; NOD, third stem internode; LOD, isolate lodicule dissected from inflorescences; LEM, lemma dissected from inflorescences; PAL, palea dissected inflorescences; CAR, whole developing grain (caryopsis, 5 and 15 days post-anthesis); SEN, senescing leaf. Detailed information is presented in Supplementary File 8 and Table S2.

\section{Conclusions}

In conclusion, $24 \mathrm{HvXTH}$ genes (named as HvXTH1-24) were identified and could be divided into three phylogenetic groups. Similar to other Poales [7], one EG16 gene was also found. Gene location analysis showed that HvXTHs are distributed unevenly on 7 barley chromosomes. HvXTH17/18 and HvXTH19/20 gene pairs may be tandem duplicates. Structure-based alignment showed that all 
HvXTHs contain conserved domains and $N$-glycosylation sites. The high expression of HvXTH8, HvXTH10, HvXTH14, HvXTH17, HvXTH18, HvXTH20 and HvXTH21 in all 16 tissues during different developmental stages suggests that $\mathrm{HvXTHs}$ might have different functions and be involved in the plant development. Taken together, based on a genome-wide identification and analysis of the $\mathrm{HvXTH}$ gene family in barley, our research provides a foundation for further functional research of $\mathrm{HvXTHs}$ in barley development.

\section{Materials and Methods}

\subsection{Identification of XTH Family Genes in H. vulgare L.}

Gene, cDNA and protein sequences were retrieved from the Ensembl Plants database (http: //plants.ensembl.org) [46]. Two methods were used to identify barley XTH proteins in this study. Firstly, the Hidden Markov Model (HMM) was used to search the protein database using HMMER3.0 with default parameters in which the profiles of the XTH protein domains, PF00722 and PF06955, were used as queries. The online program SMART (http://smart.embl-heidelberg.de/) [47] was used to identify conserved domains of candidate barley XTHs. Only the proteins containing both domains were kept for further analysis. The second method was based on the BLAST analysis of homologous proteins. Twenty-nine published OsXTH protein sequences [10] were used as queries to map the $H$. vulgare genome database using the parameters E-value $<10^{-15}$ and identity $>50 \%$ and redundant sequences were removed manually. Then, the remaining candidate XTH protein sequences with conserved PF00722 and PF06955 domains were filtered by the SMART tool using an E-value of $<0.1$. After that, the gene structure was checked according to information of IPK and sequences published in NCBI. Some ORFs were rejected because they contained uncharacteristic amino acid sequences located in the predicted C-terminal regions or lacked the signature motif that includes the residues responsible for the catalytic activity and is conserved among all the XTHs thus far characterized [8,34]. In these cases, the coding regions of these predicted genes were manually reinterpreted based both on the conserved structural features of XTHs and on the sequence database of full-length cDNAs. Some annotation of HvXTH CDS sequences was not consistent with the NCBI BLAST analysis, so we compared the data in IPK and NCBI and reannotated them. Finally, XTH proteins of barley were identified based on methods described above and used for further analysis. The names of barley XTH genes were nominated according to the previous study [34].

\subsection{Phylogenetic Analysis of HvXTH Genes}

Phylogenetic analysis was performed using the deduced amino acid sequences obtained with identified HvXTHs together with 29 OsXTH genes and 33 AtXTH genes; the structurally characterized bacterial lichenase, 1GBG; was used as an outgroup. After removing the predicted signal peptide, alignment analysis of amino acid sequences was performed using MAFFT with the iterative refinement method and the scoring matrix Blosum62 [48]. Then the obtained alignment was manually refined by Bioedit (http://www.mbio.ncsu.edu/bioedit/bioedit.html), based on the crystal structures of Ptt-XET16-34 and Tm-NXG1. A phylogenetic tree was constructed using maximum likelihood (ML) by PhyML [49]. The percentages of trees in which the associated taxa clustered together were determined from 100 bootstrap replications.

\subsection{Gene Structure Analysis and Chromosomal Location}

The Gene Structure Display Server (GSDS) tool (http://gsds.cbi.pku.edu.cn/) [50] was employed to identify exon/intron organization of the HvXTH genes by aligning the CDS sequences with the corresponding genomic DNA sequences. Putative signal peptide sequences were predicted using the online website SignalP (http://www.cbs.dtu.dk/services/SignalP/) [51]. The MEME tool (http://meme-suite.org/tools/meme) [52] was used to predict the DEIDFEFLG motif. The chromosomal 
locations of the $\mathrm{HvXTH}$ genes were physically mapped to each chromosome according to their positions in the barley genome. The physical map was depicted using MapChart tools (v2.3.2) [53].

\subsection{Structural-Based Sequence Alignment}

The online tool, ESPript (http://espript.ibcp.fr/ESPript/ESPript/) [54], was used to predict the secondary structures as well as the presence of structural elements in the HvXTH protein sequences. Alignment of the candidate HvXTH sequences was conducted by ClustalW with default settings that aimed to identify common structural elements of XTHs in barley. The crystal structure of TmNXG1 (PDB id: 2UWA) [11] and that of PttXET16-34 (PDB id:1UN1) [35] were obtained from the PDB databank to locate secondary structures.

\subsection{Gene Expression Analysis}

RNA-Seq data of 16 developmental stages published previously was downloaded from the ENA (Accession number: PRJUEB3149, PRJEB14349) database to analyze the different expression patterns of HvXTHs [29]. The data filtered by Trimmomatic were mapped to the genome using TopHat (v2.1.1) [55] with the default settings. Cufflinks (v2.2.1) was used to predict expression level of genes and is presented as fragments per kilobase of exon per million fragments mapped (FPKM) (parameters: -G -b -compatible-hits-norm -F 0.00001). Then, the expression levels of the transcript were calculated by Cuffdiff (parameters: -upper-quartile-norm, -frag-bias-correct). Finally, the expression levels of $\mathrm{HvXTHs}$ was normalized using $\log _{10}(\mathrm{FPKM}+1)$. The developmental stages analyzed were: embryonic tissue (EMB); isolate etiolated leaves (ETI); root tissue (17 days and 28 days after planting) (ROO, ROO2); leaf tissue (LEA); epidermal strips (EPI); whole developing inflorescence tissue (30 days and 50 days after planting) (INF1, INF2); rachis (RAC); third stem internodes (NOD); isolated lodicules dissected from inflorescences (LOD); lemma dissected from inflorescences (LEM); palea dissected from inflorescences (PAL); whole developing grain (caryopsis, 5 and 15 days post-anthesis) (CAR5, CAR15); senescing leaves (SEN).

Supplementary Materials: The following are available online. Supplementary File 1: Protein sequences of XTHs employed in the phylogenetic analysis (DOCX $29 \mathrm{~kb}$ ); Supplementary File 2: Coding sequences of HvXTHs (DOCX $21 \mathrm{~kb}$ ); Supplementary File 3: Genomic sequences of HvXTHs (DOCX $27 \mathrm{~kb}$ ); Supplementary File 4: Table S1. Pairwise identities between paralogous pairs of $\mathrm{HvXTH}$ genes (DOCX $16 \mathrm{~kb}$ ); Supplementary File 5: The multiple alignment of deduced amino acid sequences of HvXTHs 22-28and 2UWA (PDF $23 \mathrm{~kb}$ ); Supplementary File 6: The multiple alignment of deduced amino acid sequences of HvXTHs and 2UWA 1-21 (PDF $28 \mathrm{~kb}$ ); Supplementary File 7: The multiple alignment of deduced amino acid sequences of HvXTHs and EG16(PDF 28kb); Supplementary File 8: Table S2. Plant tissues for RNA-seq (DOCX $18 \mathrm{~kb}$ ).

Author Contributions: Conceptualization, M.-M.F. and F.W.; data curation, M.M.F. and C.L.; formal analysis, M.-M.F. and C.L.; funding acquisition, F.W.; investigation, M.-M.F.; methodology, M.-M.F.; resources, F.W.; software, C.L.; supervision, F.W.; writing—original draft, M.-M.F.; writing—review \& editing, M.-M.F. and F.W.

Funding: The Key Research Foundation of Science and Technology Department of Zhejiang Province of China (2016C02050-9-7). The funding body had no role in the design of the study, collection, analysis or interpretation of data or in writing the manuscript.

Conflicts of Interest: The authors declare no conflict of interest.

\section{References}

1. Nishitani, K.; Tominaga, R. Endoxyloglucan Transferase, a Novel Class of Glycosyltransferase That Catalyzes Transfer of a Segment of Xyloglucan Molecule to Another Xyloglucan Molecule. J. Biol. Chem. 1992, 267, 21058-21064. [PubMed]

2. Faure, R.; Saura-Valls, M.; Brumer, H., 3rd; Planas, A.; Cottaz, S.; Driguez, H. Synthesis of a library of xylogluco-oligosaccharides for active-site mapping of xyloglucan endo-transglycosylase. J. Org. Chem. 2006, 71, 5151-5161. [CrossRef] 
3. Simmons, T.J.; Mohler, K.E.; Holland, C.; Goubet, F.; Frankova, L.; Houston, D.R.; Hudson, A.D.; Meulewaeter, F.; Fry, S.C. Hetero-trans-glucanase, an enzyme unique to Equisetum plants, functionalizes cellulose. Plant J. 2015, 83, 753-769. [CrossRef] [PubMed]

4. Michel, G.; Chantalat, L.; Duee, E.; Barbeyron, T.; Henrissat, B.; Kloareg, B.; Dideberg, O. The kappa-carrageenase of P-carrageenovora features a tunnel-shaped active site: A novel insight in the evolution of clan-B glycoside hydrolases. Structure 2001, 9, 513-525. [CrossRef]

5. Eklof, J.M.; Shojania, S.; Okon, M.; McIntosh, L.P.; Brumer, H. Structure-Function Analysis of a Broad Specificity Populus trichocarpa Endo-beta-glucanase Reveals an Evolutionary Link between Bacterial Licheninases and Plant XTH Gene Products. J. Biol. Chem. 2013, 288, 15786-15799. [CrossRef]

6. McGregor, N.; Yin, V.; Tung, C.C.; Van Petegem, F.; Brumer, H. Crystallographic insight into the evolutionary origins of xyloglucan endotransglycosylases and endohydrolases. Plant J. 2017, 89, 651-670. [CrossRef]

7. Behar, H.; Graham, S.W.; Brumer, H. Comprehensive cross-genome survey and phylogeny of glycoside hydrolase family 16 members reveals the evolutionary origin of EG16 and XTH proteins in plant lineages. Plant J. 2018, 95, 1114-1128. [CrossRef]

8. Yokoyama, R.; Nishitani, K. A comprehensive expression analysis of all members of a gene family encoding cell-wall enzymes allowed us to predict cis-regulatory regions involved in cell-wall construction in specific organs of arabidopsis. Plant Cell Physiol. 2001, 42, 1025-1033. [CrossRef]

9. Campbell, P.; Braam, J. Xyloglucan endotransglycosylases: Diversity of genes, enzymes and potential wall-modifying functions. Trends Plant Sci. 1999, 4, 361-366. [CrossRef]

10. Yokoyama, R.; Rose, J.K.C.; Nishitani, K. A surprising diversity and abundance of xyloglucan endotransglucosylase/hydrolases in rice. Classification and expression analysis. Plant Physiol. 2004, 134, 1088-1099. [CrossRef]

11. Baumann, M.J.; Eklof, J.M.; Michel, G.; Kallas, A.M.; Teeri, T.T.; Czjzek, M.; Brumer, H., 3rd. Structural evidence for the evolution of xyloglucanase activity from xyloglucan endo-transglycosylases: Biological implications for cell wall metabolism. Plant Cell 2007, 19, 1947-1963. [CrossRef] [PubMed]

12. Opazo, M.C.; Lizana, R.; Stappung, Y.; Davis, T.M.; Herrera, R.; Moya-Leon, M.A. XTHs from Fragaria vesca: Genomic structure and transcriptomic analysis in ripening fruit and other tissues. BMC Genom. 2017, 18, 852. [CrossRef] [PubMed]

13. Jan, A.; Yang, G.X.; Nakamura, H.; Ichikawa, H.; Kitano, H.; Matsuoka, M.; Matsumoto, H.; Komatsu, S. Characterization of a xyloglucan endotransglucosylase gene that is up-regulated by gibberellin in rice. Plant Physiol. 2004, 136, 3670-3681. [CrossRef]

14. Osato, Y.; Yokoyama, R.; Nishitani, K. A principal role for AtXTH18 in Arabidopsis thaliana root growth: A functional analysis using RNAi plants. J. Plant Res. 2006, 119, 153-162. [CrossRef] [PubMed]

15. Keuskamp, D.H.; Sasidharan, R.; Vos, I.; Peeters, A.J.M.; Voesenek, L.A.C.J.; Pierik, R. Blue-light-mediated shade avoidance requires combined auxin and brassinosteroid action in Arabidopsis seedlings. Plant J. 2011, 67, 208-217. [CrossRef]

16. Pitaksaringkarn, W.; Matsuoka, K.; Asahina, M.; Miura, K.; Sage-Ono, K.; Ono, M.; Yokoyama, R.; Nishitani, K.; Ishii, T.; Iwai, H.; et al. XTH20 and XTH19 regulated by ANAC071 under auxin flow are involved in cell proliferation in incised Arabidopsis inflorescence stems. Plant J. 2014, 80, 604-614. [CrossRef]

17. Han, Y.; Ban, Q.; Hou, Y.; Meng, K.; Suo, J.; Rao, J. Isolation and Characterization of Two Persimmon Xyloglucan Endotransglycosylase/Hydrolase (XTH) Genes That Have Divergent Functions in Cell Wall Modification and Fruit Postharvest Softening. Front. Plant Sci. 2016, 7. [CrossRef]

18. Liu, Y.; Liu, D.; Zhang, H.; Gao, H.; Guo, X.; Wang, D.; Zhang, X.; Zhang, A. The alpha- and beta-expansin and xyloglucan endotransglucosylase/hydrolase gene families of wheat: Molecular cloning, gene expression and EST data mining. Genomics 2007, 90, 516-529. [CrossRef]

19. Rai, K.M.; Thu, S.W.; Balasubramanian, V.K.; Cobos, C.J.; Disasa, T.; Mendu, V. Identification, Characterization and Expression Analysis of Cell Wall Related Genes in Sorghum bicolor (L.) Moench, a Food, Fodder and Biofuel Crop. Front. Plant Sci. 2016, 7, 1287. [CrossRef]

20. Wang, M.; Xu, Z.; Ding, A.; Kong, Y. Genome-Wide Identification and Expression Profiling Analysis of the Xyloglucan Endotransglucosylase/Hydrolase Gene Family in Tobacco (Nicotiana tabacum L.). Genes 2018, 9 , 273. [CrossRef]

21. Song, L.; Valliyodan, B.; Prince, S.; Wan, J.; Nguyen, H.T. Characterization of the XTH Gene Family: New Insight to the Roles in Soybean Flooding Tolerance. Int. J. Mol. Sci. 2018, 19, 2705. [CrossRef] 
22. Saladie, M.; Rose, J.K.; Cosgrove, D.J.; Catala, C. Characterization of a new xyloglucan endotransglucosylase/hydrolase (XTH) from ripening tomato fruit and implications for the diverse modes of enzymic action. Plant J. 2006, 47, 282-295. [CrossRef] [PubMed]

23. Miedes, E.; Lorences, EP. Xyloglucan endotransglucosylase/hydrolases (XTHs) during tomato fruit growth and ripening. J. Plant Physiol. 2009, 166, 489-498. [CrossRef]

24. Geisler-Lee, J.; Geisler, M.; Coutinho, P.M.; Segerman, B.; Nishikubo, N.; Takahashi, J.; Aspeborg, H.; Djerbi, S.; Master, E.; Andersson-Gunneras, S.; et al. Poplar carbohydrate-active enzymes. Gene identification and expression analyses. Plant Physiol. 2006, 140, 946-962. [CrossRef]

25. Purugganan, M.D.; Fuller, D.Q. The nature of selection during plant domestication. Nature 2009, 457, 843-848. [CrossRef]

26. Forster, B.P.; Ellis, R.P.; Thomas, W.T.; Newton, A.C.; Tuberosa, R.; This, D.; el-Enein, R.A.; Bahri, M.H.; Ben Salem, M. The development and application of molecular markers for abiotic stress tolerance in barley. J. Exp. Bot. 2000, 51, 19-27. [CrossRef]

27. Schunmann, P.H.D.; Smith, R.C.; Lang, V.; Matthews, P.R.; Chandler, PM. Expression of XET-related genes and its relation to elongation in leaves of barley (Hordeum vulgare L.). Plant Cell Environ. 1997, 20, 1439-1450. [CrossRef]

28. Kaewthai, N.; Harvey, A.J.; Hrmova, M.; Brumer, H.; Ezcurra, I.; Teeri, T.T.; Fincher, G.B. Heterologous expression of diverse barley XTH genes in the yeast Pichia pastoris. Plant Biotechnol. J. 2010, 27, 251-258. [CrossRef]

29. Mascher, M.; Gundlach, H.; Himmelbach, A.; Beier, S.; Twardziok, S.O.; Wicker, T.; Radchuk, V.; Dockter, C.; Hedley, P.E.; Russell, J.; et al. A chromosome conformation capture ordered sequence of the barley genome. Nature 2017, 544, 427-433. [CrossRef]

30. Mayer, K.F.X.; Waugh, R.; Langridge, P.; Close, T.J.; Wise, R.P.; Graner, A.; Matsumoto, T.; Sato, K.; Schulman, A.; Muehlbauer, G.J.; et al. A physical, genetic and functional sequence assembly of the barley genome. Nature 2012, 491, 711. [PubMed]

31. Hrmova, M.; Farkas, V.; Harvey, A.J.; Lahnstein, J.; Wischmann, B.; Kaewthai, N.; Ezcurra, I.; Teeri, T.T.; Fincher, G.B. Substrate specificity and catalytic mechanism of a xyloglucan xyloglucosyl transferase HvXET6 from barley (Hordeum vulgare L.). FEBS J. 2009, 276, 437-456. [CrossRef]

32. Kaewthai, N.; Gendre, D.; Eklof, J.M.; Ibatullin, F.M.; Ezcurra, I.; Bhalerao, R.P.; Brumer, H. Group III-A XTH Genes of Arabidopsis Encode Predominant Xyloglucan Endohydrolases That Are Dispensable for Normal Growth. Plant Physiol. 2013, 161, 440-454. [CrossRef]

33. Zhu, X.F.; Shi, Y.Z.; Lei, G.J.; Fry, S.C.; Zhang, B.C.; Zhou, Y.H.; Braam, J.; Jiang, T.; Xu, X.Y.; Mao, C.Z.; et al. XTH31, Encoding an in Vitro XEH/XET-Active Enzyme, Regulates Aluminum Sensitivity by Modulating in Vivo XET Action, Cell Wall Xyloglucan Content and Aluminum Binding Capacity in Arabidopsis. Plant Cell 2012, 24, 4731-4747. [CrossRef]

34. Rose, J.K.C.; Braam, J.; Fry, S.C.; Nishitani, K. The XTH family of enzymes involved in xyloglucan endotransglucosylation and endohydrolysis: Current perspectives and a new unifying nomenclature. Plant Cell Physiol. 2002, 43, 1421-1435. [CrossRef]

35. Johansson, P.; Brumer, H., 3rd; Baumann, M.J.; Kallas, A.M.; Henriksson, H.; Denman, S.E.; Teeri, T.T.; Jones, T.A. Crystal structures of a poplar xyloglucan endotransglycosylase reveal details of transglycosylation acceptor binding. Plant Cell 2004, 16, 874-886. [CrossRef]

36. Henriksson, H.; Denman, S.E.; Campuzano, I.D.G.; Ademark, P.; Master, E.R.; Teeri, T.T.; Brumer, H. N-linked glycosylation of native and recombinant cauliflower xyloglucan endotransglycosylase 16A. Biochem. J. 2003, 375, 61-73. [CrossRef]

37. Schroder, R.; Atkinson, R.G.; Langenkamper, G.; Redgwell, R.J. Biochemical and molecular characterisation of xyloglucan endotransglycosylase from ripe kiwifruit. Planta 1998, 204, 242-251. [CrossRef]

38. Campbell, P.; Braam, J. In vitro activities of four xyloglucan endotransglycosylases from Arabidopsis. Plant J. 1999, 18, 371-382. [CrossRef]

39. Becnel, J.; Natarajan, M.; Kipp, A.; Braam, J. Developmental expression patterns of Arabidopsis XTH genes reported by transgenes and Genevestigator. Plant Mol. Biol. 2006, 61, 451-467. [CrossRef]

40. Aubert, D.; Herzog, M. A new cDNA encoding a xyloglucan endo-transglycosylase-related polypeptide (AtXTR8) preferentially expressed in seedling, root and stem of Arabidopsis thaliana. Plant Sci. 1996, 121, 187-196. [CrossRef] 
41. Catala, C.; Rose, J.K.; Bennett, A.B. Auxin regulation and spatial localization of an endo-1,4- $\beta$-D-glucanase and a xyloglucan endotransglycosylase in expanding tomato hypocotyls. Plant J. 1997, 12, 417-426. [CrossRef]

42. Smith, R.C.; Matthews, P.R.; Schunmann, P.H.D.; Chandler, P.M. The regulation of leaf elongation and xyloglucan endotransglycosylase by gibberellin in 'Himalaya' barley (Hordeum vulgare L.). J. Exp. Bot. 1996, 47, 1395-1404. [CrossRef]

43. Barrachina, C.; Lorences, EP. Xyloglucan endotransglycosylase activity in pine hypocotyls. Intracellular localization and relationship with endogenous growth. Physiol. Plantarum 1998, 102, 55-60. [CrossRef]

44. Gerttula, S.; Zinkgraf, M.; Muday, G.K.; Lewis, D.R.; Ibatullin, F.M.; Brumer, H.; Hart, F.; Mansfield, S.D.; Filkov, V.; Groover, A. Transcriptional and Hormonal Regulation of Gravitropism of Woody Stems in Populus. Plant Cell 2015, 27, 2800-2813. [PubMed]

45. Mellerowicz, E.J.; Immerzeel, P.; Hayashi, T. Xyloglucan: The Molecular Muscle of Trees. Ann. Bot. 2008, 102, 659-665. [CrossRef]

46. Kersey, P.J.; Allen, J.E.; Allot, A.; Barba, M.; Boddu, S.; Bolt, B.J.; Carvalho-Silva, D.; Christensen, M.; Davis, P.; Grabmueller, C.; et al. Ensembl Genomes 2018: An integrated omics infrastructure for non-vertebrate species. Nucleic Acids Res. 2018, 46, D802-D808. [CrossRef] [PubMed]

47. Letunic, I.; Bork, P. 20 years of the SMART protein domain annotation resource. Nucleic Acids Res. 2018, 46, D493-D496. [CrossRef]

48. Katoh, K.; Misawa, K.; Kuma, K.; Miyata, T. MAFFT: A novel method for rapid multiple sequence alignment based on fast Fourier transform. Nucleic Acids Res. 2002, 30, 3059-3066. [CrossRef] [PubMed]

49. Guindon, S.; Gascuel, O. A simple, fast and accurate algorithm to estimate large phylogenies by maximum likelihood. Systematic Biol. 2003, 52, 696-704. [CrossRef]

50. Hu, B.; Jin, J.P.; Guo, A.Y.; Zhang, H.; Luo, J.C.; Gao, G. GSDS 2.0: An upgraded gene feature visualization server. Bioinformatics 2015, 31, 1296-1297. [CrossRef]

51. Armenteros, J.J.A.; Tsirigos, K.D.; Sonderby, C.K.; Petersen, T.N.; Winther, O.; Brunak, S.; von Heijne, G.; Nielsen, H. SignalP 5.0 improves signal peptide predictions using deep neural networks. Nat. Biotechnol. 2019, 37, 420. [CrossRef] [PubMed]

52. Bailey, T.L.; Boden, M.; Buske, F.A.; Frith, M.; Grant, C.E.; Clementi, L.; Ren, J.Y.; Li, W.W.; Noble, W.S. MEME SUITE: Tools for motif discovery and searching. Nucleic Acids Res. 2009, 37, W202-W208. [CrossRef]

53. Voorrips, R.E. MapChart: Software for the graphical presentation of linkage maps and QTLs. J. Hered. 2002, 93, 77-78. [CrossRef]

54. Robert, X.; Gouet, P. Deciphering key features in protein structures with the new ENDscript server. Nucleic Acids Res. 2014, 42, W320-W324. [CrossRef] [PubMed]

55. Kim, D.; Pertea, G.; Trapnell, C.; Pimentel, H.; Kelley, R.; Salzberg, S.L. TopHat2: Accurate alignment of transcriptomes in the presence of insertions, deletions and gene fusions. Genome Biol. 2013, 14. [CrossRef] [PubMed]

Sample Availability: Not available.

(C) 2019 by the authors. Licensee MDPI, Basel, Switzerland. This article is an open access article distributed under the terms and conditions of the Creative Commons Attribution (CC BY) license (http://creativecommons.org/licenses/by/4.0/). 